\title{
Low power compact radio galaxies at high angular resolution
}

\author{
M. Giroletti ${ }^{1,2}$, G. Giovannini ${ }^{1,2}$, and G. B. Taylor ${ }^{3,4}$ \\ 1 Dipartimento di Astronomia, Università di Bologna, via Ranzani 1, 40127 Bologna, Italy \\ e-mail: [giroletti;ggiovann]@ira.cnr.it \\ 2 Istituto di Radioastronomia, via Gobetti 101, 40129, Bologna, Italy \\ 3 Kavli Institute of Particle Astrophysics and Cosmology, Menlo Park, CA 94025, USA \\ e-mail: gtaylor@nrao.edu \\ ${ }^{4}$ National Radio Astronomy Observatory, PO Box O, Socorro, NM 87801, USA
}

Received 2 May 2005 / Accepted 15 June 2005

\begin{abstract}
We present sub-arcsecond resolution multi-frequency ( 8 and $22 \mathrm{GHz}$ ) VLA images of five low power compact (LPC) radio sources, and phase referenced VLBA images at $1.6 \mathrm{GHz}$ of their nuclear regions. At the VLA resolution we resolve the structure and identify component positions and flux densities. The phase referenced VLBA data at $1.6 \mathrm{GHz}$ reveals flat-spectrum, compact cores (down to a few milliJansky) in four of the five sources. The absolute astrometry provided by the phase referencing allows us to identify the center of activity on the VLA images. Moreover, these data reveal rich structures, including two-sided jets and secondary components. On the basis of the arcsecond scale structures and of the nuclear properties, we rule out the presence of strong relativistic effects in our LPCs, which must be intrinsically small (deprojected linear sizes $\lesssim 10 \mathrm{kpc}$ ). Fits of continuous injection models reveal break frequencies in the $\mathrm{GHz}$ domain, and ages in the range $10^{5}-10^{7} \mathrm{yrs}$. In LPCs, the outermost edge may be advancing more slowly than in more powerful sources or could even be stationary; some LPCs might also have ceased their activity. In general, the properties of LPCs can be related to a number of reasons, including, but not limited to: youth, frustration, low kinematic power jets, and short-lived activity in the radio.
\end{abstract}

Key words. radio continuum: galaxies - galaxies: individual: $1855+37$ - galaxies: active - galaxies: jets

\section{Introduction}

Radio sources come in different well known and understood morphologies. Extended (kiloparsec scale) radio galaxies are divided between FR I and FR II radio galaxies, according to their morphology and radio power (Fanaroff \& Riley 1974). Ledlow \& Owen (1996) showed that the separation between these two classes is related to both the total radio power and the optical magnitude of the host galaxy. Ghisellini \& Celotti (2001) showed how this separation can be interpreted in terms of accretion rates below or above a critical upper limit for maintaining an optically thin advection dominated accretion flow (ADAF, Narayan \& Yi 1995) regime.

Compact (sub-kiloparsec) radio sources are also divided on the basis of different morphologies. Some of them appear as compact radio sources because of projection effects; this includes BL Lacertae objects (e.g., Mkn 501, Giroletti et al. 2004) and flat spectrum radio quasars (FSRQ), i.e., sources oriented at small angles with respect to the line of sight. Their compactness is due to projection effects, and to strong relativistic effects which give rise to one-sidedness, superluminal motions, and high brightness temperatures.

Other high power radio sources appear intrinsically small and are not affected by relativistic effects, since we do not see beamed relativistic jets but simply the regions of interaction between jets and the ISM (hot spots), which are advancing at velocities of the order of $0.2 \mathrm{c}$ (Owsianik \& Conway 1998; Polatidis et al. 2002; Giroletti et al. 2003). The Compact Symmetric Objects class (CSOs, see e.g. Wilkinson et al. 1994; Gugliucci et al. 2005) is a good example of this population: it is composed of small sources $(<1 \mathrm{kpc})$, with emission on both sides of the central engine. On the basis of kinematics as well as spectral arguments, these objects are interpreted as young radio galaxies with ages $\lesssim 10^{4}$ years and are expected to evolve into kiloparsec scale radio galaxies (Owsianik \& Conway 1998; Murgia et al. 1999; Giroletti et al. 2003). The number of these sources appears, however, to be too high with respect to the general population of radio galaxies (Fanti et al. 1990; O'Dea $\&$ Baum 1997), therefore it has been suggested that their radio power decreases and/or expansion slows with time to account for the observed population of giant radio galaxies (Kaiser et al. 1997; Blundell et al. 1999; Fanti \& Fanti 2003; Lara et al. 2004).

In catalogues of radio sources selected at low frequency there is another class of radio sources whose properties are not yet well known. We have named these low power compact (LPC) radio sources. Most of these sources do not have a 
flat radio spectra and show a moderately steep spectral index. Moreover, their host galaxies do not show signatures of strong nuclear activity in the optical and X-ray bands. X-ray luminosities, or upper limits, are typically of the order $10^{40} \mathrm{erg} \mathrm{s}^{-1}$ (e.g. Canosa et al. 1999). In the optical, central compact cores are seldom visible; and even when they are detected, these cores show a similar nature to the synchrotron optical cores of FR I radio galaxies rather than signatures of thermal emission from an efficiently radiating accretion disk (Capetti et al. 2002). The radio powers are typically below $10^{25} \mathrm{~W} \mathrm{~Hz}^{-1}$ at $1.4 \mathrm{GHz}$. Little variability is present in the radio fluxes, although this can be accounted for by the paucity of measurements.

The small size of LPCs could be directly related to the low radio power: the central AGN has insufficient power to drive the relativistic jet out of the dense ISM present in the central regions of the host galaxy. This could be the case for sources like NGC 4278 (Giroletti et al. 2005) and may constitute the link between radio loud and radio quiet AGN such as Seyfert galaxies. However, most of these low power compact radio sources have an intrinsic radio power in the same range as that of low power giant FR I radio galaxies. In this case, the reason for the compactness of these sources is unclear. It could be again any of the previous physical reasons: geometrical-relativistic effects, low age, or frustration by a denser than average ISM. It could be that all of these effects are present, and in addition some sources may even be prematurely dying (Marecki et al. 2003; Kunert-Bajraszewska et al. 2004; Gugliucci et al. 2005).

All these possibilities are interesting: in the case of geometric-relativistic effects, we would have a population of low power BL-Lacs where relativistic jets are present in spite of the low power of the AGN; in the case of young radio sources, we could test if their number is in agreement with current populations and discuss whether they will become giant radio galaxies or not. The LPC sources could be related to CSOs, but at lower radio power with respect to most CSOs studied up to now. Finally, frustration has been excluded as an explanation to the origin of CSOs, but could apply in some LPC sources, so it is important to measure kinematic ages for symmetric LPC sources.

To investigate these points we have selected from the Bologna Complete Sample (BCS, Giovannini et al. 2005) compact $(<10 \mathrm{kpc})$ radio sources with a total low frequency radio power $P_{408 \mathrm{MHz}}<10^{25.5} \mathrm{~W} \mathrm{~Hz}^{-1}$ whose sub-parsec structure was not well defined. We are studying their properties with Very Long Baseline Array (VLBA) and high frequency, high resolution Very Large Array (VLA) observations. Two sources fulfilling the same requirements (the CSO 4C 31.04 and NGC 4278) have been already discussed in published papers (Giroletti et al. 2003, 2005). Here we present new results for 5 radio galaxies: $0222+36,0258+35,0648+27,1037+30$, and $1855+37$ (see Table 1). The remaining compact sources will be observed in the near future and discussed in light of the statistical properties of the BCS.

The paper is laid out as follows: in Sect. 2 we give the details of our observations, in Sect. 3 we present the results about single sources, and in Sect. 4 we discuss the properties of our subsample with respect to other classes of compact sources; we present our main conclusions in Sect. 5. Throughout this
Table 1. Sources basic parameters

\begin{tabular}{cccc}
\hline \hline Source & $z$ & $\begin{array}{c}\log P_{408 \mathrm{MHz}} \\
\left(\mathrm{W} \mathrm{Hz}^{-1}\right)\end{array}$ & $\begin{array}{c}M_{V} \\
(\mathrm{mag})\end{array}$ \\
\hline $0222+36$ & 0.0334 & 23.91 & -22.3 \\
$0258+35$ & 0.0165 & 24.37 & -21.8 \\
$0648+27$ & 0.0414 & 24.02 & -23.3 \\
$1037+30$ & 0.0911 & 25.37 & -21.7 \\
$1855+37$ & 0.0552 & 24.65 & -23.8 \\
\hline
\end{tabular}

paper, we make use of $H_{0}=70 \mathrm{~km} \mathrm{~s}^{-1} \mathrm{Mpc}^{-1}, \Omega_{\mathrm{M}}=0.3$ and $\Omega_{\Lambda}=0.7$. Spectral indices are defined such that $S(v) \propto v^{-\alpha}$.

\section{Observations and data reduction}

\subsection{VLA observations}

VLA observations of five objects $(0222+36,0258+35$, $0648+27,1037+30$, and $1855+37$ ) were obtained in three observing runs in 2003 June 28 and 29 and 2003 July 10. The array was in "A" configuration (maximum baseline $35.4 \mathrm{~km}$ ) and the observing frequencies were 8.4 and $22 \mathrm{GHz}$. Standard observing schedules for high frequency observations were prepared, including scans to determine the primary reference pointing, and using a short ( $3 \mathrm{~s})$ integration time and fast switching mode (180 s on source, $60 \mathrm{~s}$ on calibrator) for $K$ band $(22 \mathrm{GHz})$ scans. Primary, amplitude, and phase calibrators for each run and for each source are given in Table 2 . Post-correlation processing and imaging were performed with the NRAO Astronomical Image Processing System (AIPS). Parameters of different images are reported in Table 3.

\subsection{VLBA observations}

The 5 sources are also part of a sample of 15 objects that have been observed in phase reference mode with the VLBA, in order to study the parsec scale structure of faint radio galaxies. Observations were done in two separate runs on 2003 August 07 (BG136A, $24 \mathrm{~h}$ for 12 sources) and 2003 August 30 (BG136B, 6 hrs for 3 sources). We discuss here data for the 5 LPC sources only; they have all been observed in segment A of the experiment; the VLBA structure for the other 10 sources will be discussed in a future paper.

We observed in full polarization (RCP and LCP) with two IFs (central frequency 1659.49 MHz and 1667.49 MHz). We recorded 16 channels per frequency, for a total aggregate bit rate of $128 \mathrm{Mbs}$. Each pointing on a target source was bracketed by a calibrator scan in a $5 \mathrm{~min}$ duty cycle ( $3 \mathrm{~min}$ on source, 2 min on the calibrator). Two groups of (typically) 11 cycles were executed for each source at different hour angles, resulting in a total of about $66 \mathrm{~min}$ per target, with good coverage of the $(u, v)$-plane. Calibrators were chosen from the VLBA calibrators list to be bright and close to the source; we report in Table 4 the list of the selected calibrator and its separation for each source discussed here. Short scans on strong sources (4C 39.25, J0237+2848) were interspersed with the targets and the calibrators as fringe finder sources. 
Table 2. Log of VLA observations.

\begin{tabular}{|c|c|c|c|c|c|c|c|}
\hline \multicolumn{3}{|c|}{ Absolute } & \multicolumn{2}{|c|}{$8.4 \mathrm{GHz}$} & \multicolumn{3}{|c|}{$22 \mathrm{GHz}$} \\
\hline Date & $\begin{array}{l}\text { flux density } \\
\text { calibrator }\end{array}$ & Target & $\begin{array}{c}\text { minutes } \\
\text { on source }\end{array}$ & $\begin{array}{l}\text { phase and amp. } \\
\text { calibrator }\end{array}$ & $\begin{array}{l}\text { minutes } \\
\text { on source }\end{array}$ & $\begin{array}{l}\text { amplitude } \\
\text { calibrator }\end{array}$ & $\begin{array}{c}\text { phase } \\
\text { calibrator }\end{array}$ \\
\hline \multirow[t]{3}{*}{$28 / 6$} & 3C 147 & $0648+27$ & 11 & $0645+213$ & 18 & $0741+312$ & $0657+243$ \\
\hline & $3 \mathrm{C} 48$ & $0222+36$ & 11 & $0237+288$ & 18 & $0237+288$ & $0230+405$ \\
\hline & & $0258+35$ & 11 & $0237+288$ & 18 & $0237+288$ & $0310+382$ \\
\hline $29 / 6$ & $3 \mathrm{C} 286$ & $1037+30$ & 9.5 & $0956+252$ & 12 & $0956+252$ & $1037+285$ \\
\hline $3 / 7$ & $3 \mathrm{C} 286$ & $1855+37$ & 10.5 & $1800+388$ & 18 & $1800+388$ & $1912+376$ \\
\hline
\end{tabular}

Table 3. Observational parameters.

\begin{tabular}{|c|c|c|c|c|c|c|c|c|c|}
\hline \multirow[b]{2}{*}{ Name } & \multicolumn{3}{|c|}{ VLA@8.4 GHz } & \multicolumn{3}{|c|}{ VLA @22 GHz } & \multicolumn{3}{|c|}{ VLBA@1.6 GHz } \\
\hline & $\begin{array}{c}\text { beam } \\
\left({ }^{\prime \prime} \times{ }^{\prime \prime},{ }^{\circ}\right)\end{array}$ & $\begin{array}{l}\text { noise } \\
\text { (mJy }\end{array}$ & $\begin{array}{r}\text { peak } \\
\left.\mathrm{am}^{-1}\right)\end{array}$ & $\begin{array}{c}\text { beam } \\
\left({ }^{\prime \prime} \times{ }^{\prime \prime},{ }^{\circ}\right)\end{array}$ & $\begin{array}{l}\text { noise } \\
\text { (mJy }\end{array}$ & in & $\begin{array}{c}\text { beam } \\
\left(\text { mas } \times \text { mas, }{ }^{\circ}\right)\end{array}$ & $\begin{array}{l}\text { noise } \\
\text { (mJy }\end{array}$ & peak \\
\hline $0222+36$ & $23 \times 0.19,-52$ & 0.19 & 164 & $0.11 \times 0.10,89$ & 0.17 & 89 & $10.5 \times 4.4,9$ & 0.33 & 61.2 \\
\hline $0258+35$ & $0.22 \times 0.19,-39$ & 0.21 & 126 & $0.12 \times 0.12,0$ & 0.19 & 56 & $10.4 \times 4.3,6$ & 40 & 7.5 \\
\hline $0648+27$ & $0.27 \times 0.24,65$ & 0.16 & 11.5 & $0.12 \times 0.10,-76$ & 0.19 & 2.3 & $10.6 \times 4.4,13$ & 0.57 & 4.4 \\
\hline $1037+30$ & $21 \times 0.20,9$ & 0.09 & 15.9 & $0.08 \times 0.08,15$ & 0.37 & 2.1 & $10.2 \times 5.1,22$ & 0.55 & 2.3 \\
\hline $1855+37$ & $0.27 \times 0.26,83$ & 0.06 & 0.62 & $0.19 \times 0.17,-88$ & 0.18 & - & $11.9 \times 5.3,-47$ & 0.38 & - \\
\hline
\end{tabular}

Note - Observational parameters for VLA and VLBA images in Figs. 1, 4-6, 8: beam size, lowest contour (set equal to the $3 \sigma$ noise levels) and peak flux densities. $1855+37$ is not detected with the VLA at $22 \mathrm{GHz}$ nor at $1.6 \mathrm{GHz}$ with the VLBA.

Table 4. VLBA observations: source and calibrators list.

\begin{tabular}{ccccc}
\hline \hline Source & \multicolumn{2}{c}{ Core absolute position } \\
& $\left(\mathrm{RA},{ }^{\mathrm{h} \mathrm{m} s}\right)$ & Calibrator & $\begin{array}{c}\text { Separation } \\
\left({ }^{\circ}{ }^{\circ}{ }^{\prime \prime}\right)\end{array}$ \\
\hline $0222+36$ & 022527.327 & 371027.746 & $\mathrm{~J} 0226+3421$ & 2.8 \\
$0258+35$ & 030142.329 & 351220.298 & $\mathrm{~J} 0310+3814$ & 3.8 \\
$0648+27$ & 065202.517 & 272739.405 & $\mathrm{~J} 0646+3041$ & 3.5 \\
$1037+30$ & 104029.945 & 295757.791 & $\mathrm{~J} 1037+2834$ & 1.5 \\
$1855+37$ & 185737.52 & 380033.7 & $\mathrm{~J} 1912+3740$ & 3.7 \\
\hline
\end{tabular}

Note - Incomplete coordinates for $1855+37$ are due to the lack of a detection in the VLBA and VLA $22 \mathrm{GHz}$ data. Therefore, the absolute position reported here is taken from the VLA data at $8.4 \mathrm{GHz}$.

The correlation was performed in Socorro and the initial calibrations were done with AIPS. Scans on J0237+2848 were used to remove IF-dependent delays and phase offsets. After applying models for the electron content of the ionosphere as measured by the Jet Propulsion Laboratory (JPL) on the observing dates, we performed a global fringe fitting on all calibrators and applied the solutions to the targets with a two-point interpolation. $R-L$ delay differences were determined and removed with the VLBACPOL procedure using 4C 39.25. We then produced images of the calibrators in order to obtain and apply more accurate phase and gain corrections to the sources; we also determined from preliminary maps the absolute position of the sources, which we used thereafter. We give the coordinates of the core candidate of each source in Table 4.

The final calibrated single-source datasets were imported into Difmap for imaging and self-calibration. We produced images with natural and uniform weights, and summarize in Table 3 the significant parameters for the final images.

\section{Results}

All sources are detected in our VLA data at $8.4 \mathrm{GHz}$. The source structures are successfully resolved, allowing a study of different components. At $22 \mathrm{GHz}$, we have high signal to noise detections for $0222+36,0258+35$, and $0648+27$; in the source $1037+30$ we detect only the core and N-W hot spots; finally, $1855+37$ is completely resolved and not even a faint nuclear source was detected. In sources $0222+36,0258+35$, and $0648+27$ the lack of short baselines in $22 \mathrm{GHz}$ images does not affect flux density measurements, therefore a spectral index comparison between 8.4 and $22 \mathrm{GHz}$ is meaningful. We give in Table 5 the main parameters for different sources and subcomponents, such as flux densities and spectra.

The VLBA phase referenced images in total intensity are available for all sources except $1855+37$, which is not detected. On the mas scale a resolved structure is visible in $0222+36$, $0258+35$, and $0648+27$, while in $1037+30$ we detect a hot spot in addition to the core, $\sim 0.8^{\prime \prime}$ north-west of it. Thanks to successful phase-referencing, our data yield accurate positional information which unambiguously identifies the nuclear component in these complex sources. Results are reported in Table 6, including spectral information confirming the core identification. The core spectral indices are computed between 1.6 and $22 \mathrm{GHz}$; for $0222+36$ only, we consider the total flux density detected at $1.6 \mathrm{GHz}$ by the VLBA, which extends over a region smaller than the beam of the VLA at $22 \mathrm{GHz}$. For other sources, secondary components are well separated in the VLA images $(0258+35,1037+30)$, or their flux density is negligible $(0648+27)$. We also place upper limits on the fractional polarization. In fact, all images in polarized intensity are purely noise-like, with $3 \sigma$ levels of approximately $0.25 \mathrm{mJy} / \mathrm{beam}$. Since the sources are weak, these provide weak constraints of approximately $P<10 \%$ at the peak position (except for $0222+36$, see Sect. 3.1). 
Table 5. Results of modelfit - VLA images.

\begin{tabular}{|c|c|c|c|c|c|c|c|}
\hline \multirow[b]{2}{*}{ Source } & \multirow[b]{2}{*}{ Component } & \multicolumn{2}{|c|}{ 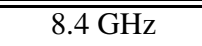 } & \multicolumn{2}{|c|}{$22.5 \mathrm{GHz}$} & \multirow[b]{2}{*}{$\alpha_{8.4}^{22}$} & \multirow[b]{2}{*}{$\begin{array}{c}\text { Size } \\
\prime \prime\end{array}$} \\
\hline & & $\begin{array}{l}\text { Peak } \\
\left(\frac{\text { mJy }}{\text { beam }}\right)\end{array}$ & $\begin{array}{r}\text { Flux } \\
(\mathrm{mJy})\end{array}$ & $\begin{array}{l}\text { Peak } \\
\left(\frac{\text { mJy }}{\text { beam }}\right)\end{array}$ & $\begin{array}{r}\text { Flux } \\
(\mathrm{mJy})\end{array}$ & & \\
\hline \multirow[t]{4}{*}{$0222+36$} & core & 174 & 179 & 89 & 91 & $0.69 \pm 0.02$ & 0.05 \\
\hline & NE lobe & 5.7 & 7.0 & 1.5 & 2.8 & $0.93 \pm 0.04$ & 0.2 \\
\hline & SW lobe & 3.2 & 5.7 & 0.6 & 2.2 & $0.97 \pm 0.04$ & 0.4 \\
\hline & total & - & 192 & - & 95 & $0.71 \pm 0.02$ & 1.6 \\
\hline \multirow[t]{5}{*}{$0258+35$} & core region & 119 & 167 & 52 & $14+57$ & $0.87 \pm 0.02$ & 0.08 \\
\hline & SE jet & 58 & 134 & 15 & 77 & $0.56 \pm 0.02$ & 1.0 \\
\hline & E lobe & 21 & 197 & 4.8 & 77 & $0.95 \pm 0.02$ & 1.6 \\
\hline & NW lobe & 14 & 93 & 2.6 & 37 & $0.93 \pm 0.02$ & 1.6 \\
\hline & total & - & 610 & - & 255 & $0.88 \pm 0.01$ & 3.5 \\
\hline \multirow[t]{3}{*}{$0648+27$} & $\mathrm{~N}$ lobe & 11 & 19 & 2.2 & $2.9+2.3$ & $1.31 \pm 0.03$ & 0.6 \\
\hline & S lobe & 8.4 & 14 & 0.9 & 4.9 & $1.06 \pm 0.03$ & 0.6 \\
\hline & total & - & 31 & - & 9.3 & $1.22 \pm 0.02$ & \\
\hline \multirow[t]{7}{*}{$1037+30$} & core & 2.2 & 3.9 & 1.0 & 2.6 & $0.41 \pm 0.06$ & 0.04 \\
\hline & jet knot & 1.2 & 2.4 & - & - & - & 0.4 \\
\hline & S lobe & 1.2 & 10 & - & - & - & 1.2 \\
\hline & S hotspot & 2.3 & 8.6 & - & - & - & 0.4 \\
\hline & $\mathrm{N}$ lobe & 5.7 & 22 & - & - & - & 0.8 \\
\hline & $\mathrm{N}$ hotspot & 13 & 20 & 4.3 & 12 & $0.52 \pm 0.02$ & 0.4 \\
\hline & total & - & 63 & - & 14 & $1.53 \pm 0.02$ & 3 \\
\hline \multirow[t]{2}{*}{$1855+37$} & core & 0.6 & 0.9 & - & - & - & 0.3 \\
\hline & total & - & 35.9 & - & - & - & 8 \\
\hline
\end{tabular}

Note - Uncertainties in Col. 7 are $1 \sigma$ values based on the absolute flux calibration and noise in the images. The core region in $0258+35$ and the northern lobe in $0648+27$ are resolved in two components at $22 \mathrm{GHz}$, therefore we give two values in Col. 6 and the spectral index is spurious. See Table 6 for a more reliable estimate of the cores spectral index. The source $1855+37$ is not detected at $22 \mathrm{GHz}$.

Table 6. Results of modelfit - VLBA images.

\begin{tabular}{llrrcc}
\hline \hline Source & Component & $\begin{array}{r}\text { Peak } \\
\left(\frac{\mathrm{mJy}}{\mathrm{beam}}\right)\end{array}$ & $\begin{array}{r}\text { Flux } \\
(\mathrm{mJy})\end{array}$ & $\begin{array}{c}\text { Size } \\
(\mathrm{mas})\end{array}$ & $\alpha_{1.6}^{22}$ \\
\hline $0222+36$ & core & 61 & 71 & 2 & $0.05 \pm 0.02$ \\
& $\mathrm{~N}$ jet & 6 & 16 & 13 & \\
& S jet & 3 & 26 & 29 & \\
& total & - & 102 & & \\
\hline $0258+35$ & core & 3.3 & 7.4 & 6 & $-0.24 \pm 0.02$ \\
& blob & 7.5 & 236 & 44 & $0.54 \pm 0.02$ \\
& total & - & 243 & & \\
\hline $0648+27$ & core & 4.3 & 10 & 5 & $0.47 \pm 0.03$ \\
& jet & 1.1 & 3.4 & 5 & \\
& total & - & 12.8 & & \\
\hline $1037+30$ & core & 3.4 & 3.8 & 5 & $0.14 \pm 0.04$ \\
& hot spot & 1.0 & 40 & 60 & $0.46 \pm 0.02$ \\
& total & - & 14 & & \\
\hline $1855+37$ & N.D. & & & \\
\hline
\end{tabular}

Note - The spectral index for $0222+36$ is computed considering the total flux density detected at $1.6 \mathrm{GHz}$ by the VLBA, which extends over a region smaller than the beam of the VLA at $22 \mathrm{GHz}$. Spectral index uncertainties are $1 \sigma$ values.

Finally, we also collected integrated flux density measurements from the NASA Extragalactic Database (NED), as well as from radio surveys including the Northern VLA Sky Survey (NVSS), the Westerbork Northern Sky Survey (WENSS), and the VLA Low-frequency Sky Survey (VLSS). These data typically cover the frequency range between $74 \mathrm{MHz}$ and $5 \mathrm{GHz}$.
Our new measurements at shorter wavelengths allow us to obtain integrated spectra spanning two and a half orders of magnitude in frequency. We show the integrated spectra for each source in the bottom right panels of Figs. 1, 4-6, 8, with bestfit continuous injection models (Murgia et al. 1999) overlaid. Detailed results for each source are presented in the following subsections.

\section{1. $0222+36$}

This source at $z=0.0334\left(1^{\prime \prime}=0.66 \mathrm{kpc}\right)$ has a flux density of $337 \mathrm{mJy}$ at $408 \mathrm{MHz}$, corresponding to a monochromatic total power of $10^{23.9} \mathrm{~W} \mathrm{~Hz}^{-1}$. Previous VLA observations at $1.4 \mathrm{GHz}$ reveal a slightly resolved morphology with a flat spectrum core surrounded by a halo extended over $\sim 8^{\prime \prime}$ (Fanti et al. 1986). On parsec scales, EVN and low resolution VLBA observations show an unresolved component with a flux density of $\sim 100 \mathrm{mJy}$ at $5 \mathrm{GHz}$ (Giovannini et al. 2001).

Our $8.4 \mathrm{GHz}$ VLA observations resolve the structure of $0222+36$ into a core and two components on either side (see Table 5). At $22 \mathrm{GHz}$ the core is the dominant structure and the two lobes are faint with an $S$ shaped structure (see Fig. 1).

The phase referenced VLBA image detects $102 \mathrm{mJy}$ of flux density at $1.6 \mathrm{GHz}$ in total intensity. No polarized flux is detected, which corresponds to a limit on the fractional polarization of $<0.4 \%$ at the core position. The source is two sided, with jets emerging in opposite directions along the north-south axis. The unresolved central core has a flux density of $58.2 \mathrm{mJy}$. 

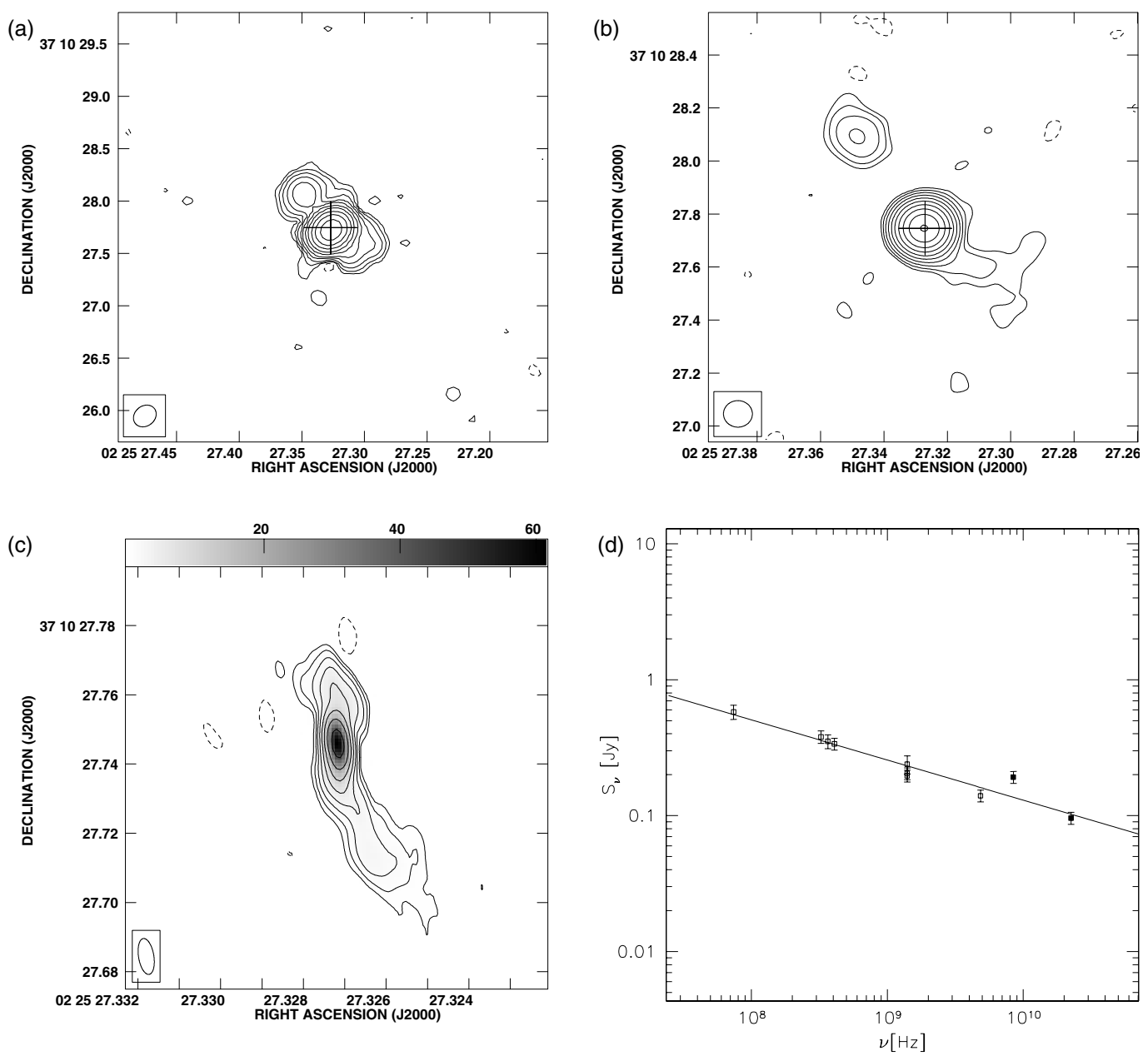

Fig. 1. Images and spectrum for $0222+36$. a) VLA at $8.4 \mathrm{GHz}$, b) VLA at $22.5 \mathrm{GHz}$, c) VLBA at $1.6 \mathrm{GHz}$, d) spectrum. Contours are traced at $(1,2,4, \ldots)$ times the $3 \sigma$ noise level; observational parameters (beam size, noise level, and peak brightness) are given in Table 3 . The cross in the VLA images indicates the position of the VLBA core. The integrated spectrum is derived from data in the present work and from the literature (see Sect. 3).

Both jets have a slightly bent path and become aligned with the kpc scale main axis at $\sim 20$ mas from the core. The resolved structure and the low core dominance are in agreement with the moderately steep spectrum of the VLA core $\left(\alpha_{8.4}^{22}=0.69 \pm 0.02\right.$; see Table 5).

Both the parsec and kiloparsec scale morphology strongly suggests that the source is oriented near the plane of the sky. At 10 mas from the peak, the jet/counter-jet brightness ratio is $R=B_{\mathrm{J}} / B_{\mathrm{CJ}}=1.3\left(B_{\mathrm{J}}=12.0 \mathrm{mJy}\right.$ and $B_{\mathrm{CJ}}=9.5 \mathrm{mJy}$, with the main jet being the one pointing north); this corresponds to $\beta \cos \theta \sim 0.05$ which implies $\theta>85^{\circ}$ if $\beta>0.6$. Therefore, the source is not affected by orientation effects and it has to be intrinsically small. If we consider the largest angular extent of the source and deproject it with an angle of $85^{\circ}$ we derive an intrinsic size of approximately $5.4 \mathrm{kpc}$.

It is intriguing to try and understand the nature of the extended radio emission surrounding the central components in the form of a $\sim 10 \mathrm{kpc}$ halo. This halo is readily visible at low frequency (Fanti et al. 1986) but it is completely resolved in our images. For this reason, we reanalyzed VLA archive data at $1.4 \mathrm{GHz}$ (D array) and $5 \mathrm{GHz}$ (in $\mathrm{A}+\mathrm{B}$ configuration). The $1.4 \mathrm{GHz} \mathrm{D}$ array data show that we are not missing a low brightness region more extended than the halo, and the $5 \mathrm{GHz}$ data allow us to derive the spectral index of the halo region. We show in Fig. 2 the $5 \mathrm{GHz} \mathrm{A}+\mathrm{B}$ array image.

From a comparison of images at different resolution (see Figs. 1 and 2), we do not see a clear connection between the inner structure (VLBA and high frequency VLA) and the extended halo; on the other hand, the VLBA structure is well connected to the source morphology as seen in the 22 and $8 \mathrm{GHz}$ images. We consider it unlikely that the halo is due to the presence of extended lobes along the line of sight because this would imply a large bend between the small and large scales with considerable fine tuning required to produce such a symmetric, uniform, and circular extended emission.

We suggest that the halo could be due to the diffusion of relativistic particles and magnetic field around the source, similar to the extended halos seen in some spiral galaxies (Hummel et al. 1991). To better investigate this point, we consider the radio spectrum of different components. In Table 7 we report flux density measurements between $74 \mathrm{MHz}$ and $22.5 \mathrm{GHz}$; the data are also shown in Fig. 3, along with a best-fit continuous injection model obtained using the Synage program (Murgia \& Fanti 1996). The total spectrum (bottom right panel of Fig. 1) 


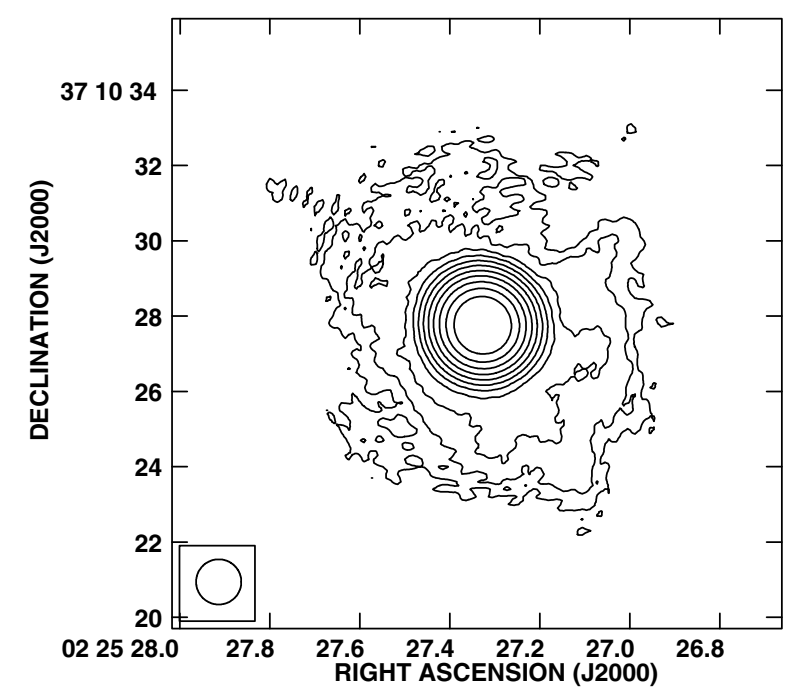

Fig. 2. Image of $0222+36$ at $5 \mathrm{GHz}$, VLA in " $\mathrm{A}+\mathrm{B}$ " configuration. Contours are traced at $(1,2,4,8, \ldots)$ times the $3 \sigma$ noise level. The lowest contour is $69 \mu \mathrm{Jy} / \mathrm{beam}$, the peak is $209 \mathrm{mJy} / \mathrm{beam}$. The beam is circular with a HPBW of $1.2^{\prime \prime}$.

Table 7. Spectral data for $0222+36$.

\begin{tabular}{lcccc}
\hline \hline $\begin{array}{l}\text { Frequency } \\
(\mathrm{GHz})\end{array}$ & $\begin{array}{c}\text { Total } \\
(\mathrm{mJy})\end{array}$ & $\begin{array}{c}\text { Halo } \\
(\mathrm{mJy})\end{array}$ & $\begin{array}{c}\text { Lobes } \\
(\mathrm{mJy})\end{array}$ & $\begin{array}{c}\text { Core } \\
(\mathrm{mJy})\end{array}$ \\
\hline $0.074(\mathrm{VLSS})$ & 580 & - & - & - \\
$0.325(\mathrm{WENSS})$ & 380 & $188^{a}$ & $191^{b}$ & $0.9^{b}$ \\
$0.365(\mathrm{TEXAS})$ & 352 & $174^{a}$ & $177^{b}$ & $1.2^{b}$ \\
$0.408(\mathrm{~B} 2)$ & 337 & $170^{a}$ & $165^{b}$ & $1.8^{b}$ \\
$1.4(\mathrm{VLA})$ & 190 & 36 & 52 & $102^{\mathrm{c}}$ \\
$5.0(\mathrm{VLA})$ & 224 & 8 & 36 & 180 \\
$8.4(\mathrm{VLA})$ & 192 & nd & 26 & 166 \\
22.5 (VLA) & 96 & nd & 6 & 90 \\
\hline
\end{tabular}

Notes $-{ }^{a}$ The flux density of the halo is computed from subtraction of the nuclear and lobe components from the total flux density listed in Col. $2 ;{ }^{b}$ lobes and core flux densities at low frequency $(0.325,0.365$, and $0.408 \mathrm{GHz}$ ) are extrapolated from data between 1.4 and $22 \mathrm{GHz}$ (see text); ${ }^{c}$ from VLBA data at $1.6 \mathrm{GHz}$.

is complex but in good agreement with the sum of spectra of different components (Fig. 3). It is also worthwhile to remember that some variability could be present and that the data at our disposal were not taken simultaneously. However, we do not find any obvious inconsistency in the data, and based on this fact we argue that the variability is insignificant.

We further point out that the core emission refers to the subarcsecond core since we do not have multi-frequency VLBI data. For this reason, at $1.6 \mathrm{GHz}$ we used the total correlated flux in our VLBA data for the core flux density. This value (102 mJy) is lower than the core flux density reported by Fanti et al. (1986), which however does definitely include a contribution from the lobes. The resulting spectrum for the sub-arcsec core shows a turnover with a maximum of $200 \mathrm{mJy}$ at $3.3 \mathrm{GHz}$. Assuming that the turnover is produced by synchrotron selfabsorption, we estimate, following Marscher (1987), the average magnetic field in the sub-arcsecond core to be in the range $2-9 \times 10^{-2}$ Gauss.
In our VLA images, both lobes show a steep spectrum between 8 and $22 \mathrm{GHz}(\alpha=0.93 \pm 0.04$ and $0.97 \pm 0.04$ in the $\mathrm{NE}$ and SW lobe, respectively). In order to obtain a better estimate of the physical parameters in the lobes, we also consider lower frequency data. These data have been obtained by subtracting the estimated flux density of the sub-arcsec core. For this reason we give in Fig. 3 and Table 7 the spectrum of the two lobes together. This explains the larger dispersion and uncertainty of this result. We estimate the average equipartition magnetic field in the two lobes with standard assumptions: we consider a frequency range from $10 \mathrm{MHz}$ to $100 \mathrm{GHz}$, an equal amount of energy in heavy particles and in electrons $(k=1)$, and that relativistic particles and magnetic fields occupy the same volume $(\phi=1)$. Under these assumptions, we find $B_{\text {eq }}=1.3 \times 10^{-4}$ Gauss in the lobes and a break frequency $v_{\mathrm{br}} \sim 9 \mathrm{GHz}$.

On larger scales, we derive a flux density of the halo from the archival data in $\mathrm{A}$ and $\mathrm{B}$ configuration at $5 \mathrm{GHz}$ (see the image obtained combining $\mathrm{A}+\mathrm{B}$ data in Fig. 2). Similarly to the case of the lobes, we derive the equipartition magnetic field in the extended halo. The spectrum of this region is straight and steep ( $\alpha \sim 1.2$ ), with a low break frequency $v_{\text {br }} \lesssim 0.3 \mathrm{GHz}$. In this region we have $B_{\mathrm{eq}} \sim 6.9 \times 10^{-6}$ Gauss.

From the spectral index information and the equipartition magnetic field, we estimate the radiative age in the lobes and in the halo region. We find that the lobes are $4.5 \times 10^{5} \mathrm{yrs}$ old and the extended halo is $\gtrsim 1.3 \times 10^{8}$ yrs old. Therefore, we have in this source a young structure (inner lobes) surrounded by an older symmetric and diffuse region. Since there is evidence of a change of the jet direction in the inner young region, we can speculate that the jet orientation is rotating because of instabilities of the AGN. This could explain the small size of the source because an unstable jet did not allow the growth of a large scale radio galaxy. In this case the old round halo could be due to the diffusion of radio emission during the orbit of the inner structure. From VLBA and high resolution VLA images we can estimate that the jet rotated by $\sim 40^{\circ}$ in $5 \times 10^{5}$ yrs so that in $10^{8}$ yrs (the halo age) it could have done many complete orbits. Higher sensitivity $\mathrm{A}+\mathrm{B}+\mathrm{C}$ data at $8.4 \mathrm{GHz}$ might be able to follow the radio structure from the lobes to the halo to test this hypothesis.

We can also compare our estimate of the spectral age of the lobes to a reasonable dynamic estimate based on their size. Assuming that the two lobes separation velocity is $\sim 0.1 \mathrm{c}$, we derive a dynamic age $\sim 2 \times 10^{4}$ yrs. This age is 10 times lower than the radiative age, but such differences have been found in other CSOs (e.g. 4C 31.04, Giroletti et al. 2003) and could be a result of the many assumptions going into both the spectral and kinematic age estimates (e.g., constant advance velocity and equipartition conditions).

\section{2. $0258+35$ (NGC 1167)}

At $z=0.0165\left(1^{\prime \prime}=0.34 \mathrm{kpc}\right)$, this source has a radio power at $408 \mathrm{MHz}$ of $10^{24.37} \mathrm{~W} \mathrm{~Hz}^{-1}$. It is optically classified as a Seyfert 2 galaxy (Ho et al. 1997), and in the radio it has been previously studied and classified as a compact steep spectrum 

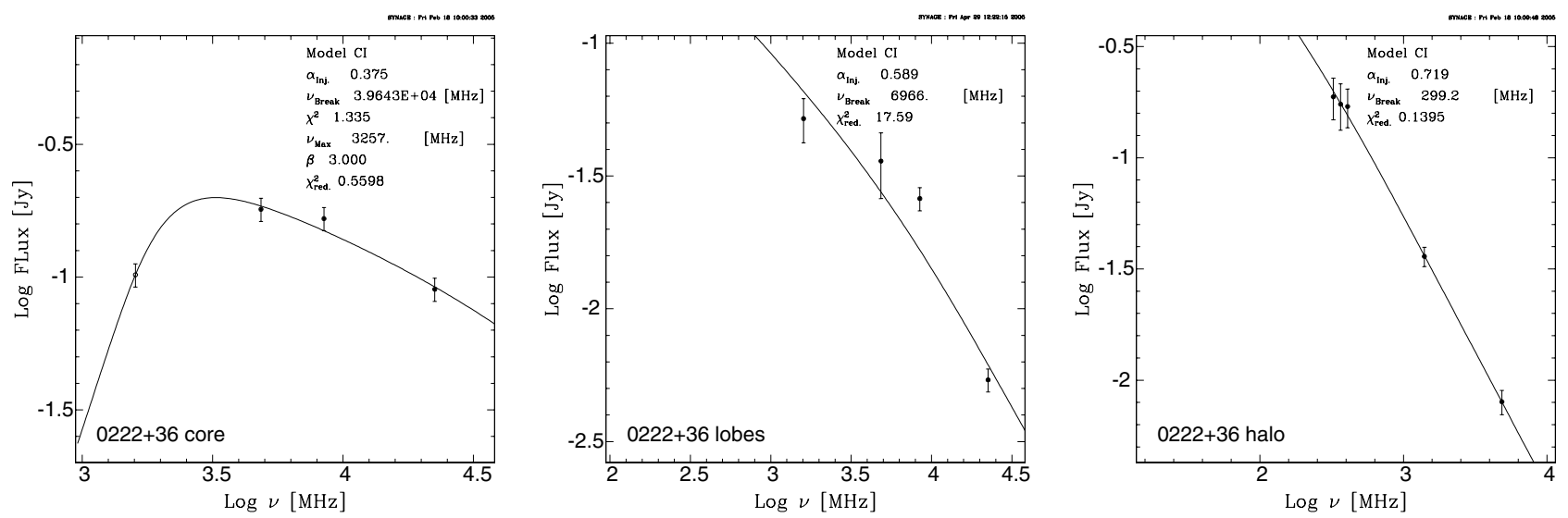

Fig. 3. Spectra for the three components of $0222+36$ : a) core, b) lobes, c) halo. The solid lines are fits with a model of continuous injection and low frequency self-absorption. The spectral points and the parameters resulting from the fits are presented in Table 7.

(CSS) source by Sanghera et al. (1995), even though the total spectrum is only moderately steep: $\alpha_{0.08}^{22}=0.54$. In contrast with most CSS sources, $0258+35$ shows plume-like lobes without prominent hot-spots. Previous studies include the global VLBI image at $5 \mathrm{GHz}$ in Giovannini et al. (2001), which reveals a compact component with a short jet-like structure and an excess of emission on the short spacings.

Our VLA images are shown in Fig. 4 (top panels). The $8.4 \mathrm{GHz}$ image shows a morphology in good agreement with previous images. Thanks to the high sensitivity of the VLA at this frequency, an extended diffuse emission surrounding the inner radio structure is detected. In the $22 \mathrm{GHz}$ image, thanks to the better angular resolution, the source peak is resolved into two components, one of which is fainter and more central, while the other is stronger and located at the beginning of the SE lobe.

Our phase-referenced VLBI data show a faint compact component identified with the nuclear source and an extended "blob" at $\sim 0.1 "$. As shown by the cross overlaid on the VLA images, the compact parsec scale core is located in the central component at the base of the south-east jet-like feature. This component is easily identifiable in the $22 \mathrm{GHz}$ image, while in the $8 \mathrm{GHz}$ map the core is confused with a bright jet component. Besides the faint compact core $\left(S_{\mathrm{c}}=7.6 \mathrm{mJy}\right)$, the largest fraction of flux density $(240 \mathrm{mJy})$ in the VLBI image at $1.6 \mathrm{GHz}$ is contained in a bright diffuse region coincident with the peak of the VLA images. The flux density and size of the components are given in Table 6. We wonder about the nature of this region because of its morphology and the lack of a connection with the parsec-scale core. It could be the result of a burst of activity of the central AGN, in which case some moderate Doppler boosting is necessary to explain the absence of a similar counterjet on the opposite side. From the jet to counter-jet ratio in the $22 \mathrm{GHz}$ VLA map measured at $\sim 0.5^{\prime \prime}$ from the core, we find that the source has to be oriented at an angle $<70^{\circ}$; if the source has subarcsecond relativistic jets, the orientation is in the range $60^{\circ} \lesssim \theta \lesssim 70^{\circ}$, in agreement with the optical classification of the parent galaxy as a Seyfert 2 . From the VLBI image and using the knot brightness we find $R=B_{\mathrm{J}} / B_{\mathrm{CJ}} \geq 7.5 / 0.13 \sim 58$, resulting in $\beta \cos \theta>0.67$, and a viewing angle $40^{\circ} \lesssim \theta \lesssim 50^{\circ}$, if $\beta \geq 0$.9. However, we cannot rule out the possibility that the knot was produced in a peculiar episode and the jet/counterjet ratio is not meaningful.

The $22 \mathrm{GHz}$ VLA image has about the same resolution ( $\sim 80$ mas) as the combined EVN+MERLIN $1.6 \mathrm{GHz}$ map shown in Sanghera et al. (1995), and we can estimate the spectral index for the core $(\alpha \sim 0.0)$ and main jet component $(\alpha \sim 0.4)$. A flat core spectral index is confirmed by our VLA images, albeit a slight misalignment between the two images prevents an unambiguous core identification only from our data. The spectrum is still flat in the central region and gradually steepens to become almost constant (0.6) in the inner bright jet-like structure in both lobes. The surrounding diffuse emission is steep: 1.0-1.5. The integrated spectral index at high frequency is $\alpha_{8.4}^{22}=0.88 \pm 0.01$.

Since the knot is well resolved in our VLBA image we derive its opening angle. We measure an angle of $26^{\circ}$, which is close to the intrinsic opening angle if the source is oriented near the plane of the sky. A free expanding jet is expected to show an intrinsic opening angle of $\sim 1 / \gamma$ (see, e.g. Salvati et al. 1998); under this assumption, we estimate a Lorentz factor $\gamma \sim 2.4$ which corresponds to $\beta \sim 0.9$.

The source structure suggests that in the data at $5 \mathrm{GHz}$ (Giovannini et al. 2001) the main peak is produced by the nuclear source with the beginning of the main jet also visible. In this case the VLBI core has an inverted spectrum, free-free or self-absorbed (26 mJy at $5 \mathrm{GHz}$ and $7.5 \mathrm{mJy}$ at $1.6 \mathrm{GHz}$ ).

We have estimated the average equipartition magnetic fields in the source, assuming an uniform brightness in the source volume. This can be considered a good approximation, since the compact structures (core and knot) are only $\sim 20 \%$ of the total flux density at $5 \mathrm{GHz}$. We estimate $B_{\text {eq }} \sim$ $9 \times 10^{-5}$ Gauss. With this estimate and using the break frequency found in the total spectral index distribution $(4.6 \mathrm{GHz})$ we estimate an age of $9 \times 10^{5} \mathrm{yrs}$ for this source. Of course, this is an average age and we expect that external diffuse regions are older with respect to the innermost region.

In light of these results, we speculate that this source might not grow to become a kiloparsec scale radio galaxy. No final hot spots demarcating the ends of the jets are visible and the source structure appears to strongly interact with the ISM as shown by the large bending of the arcsecond structure of the SE lobe and 

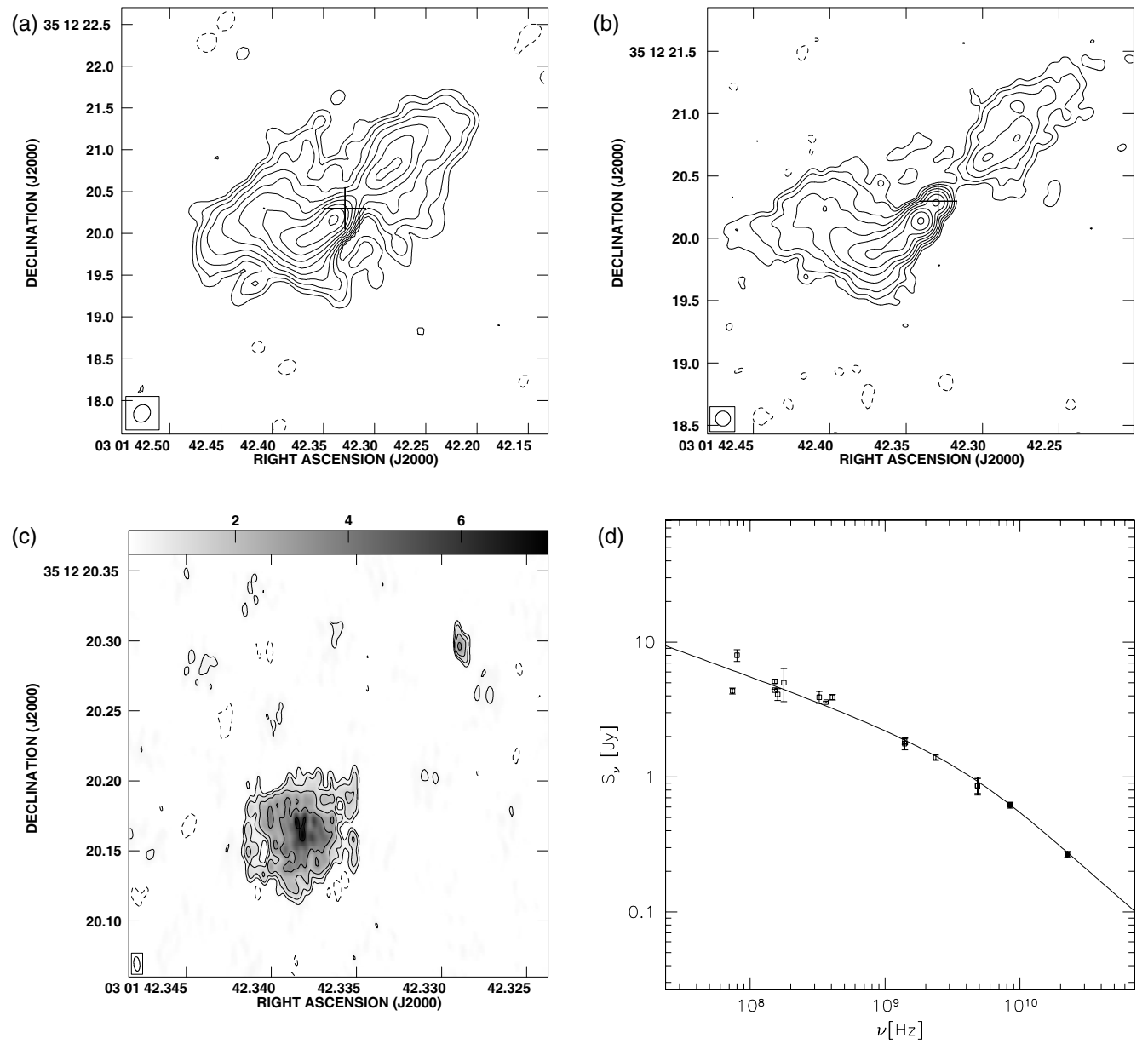

Fig. 4. Images and spectrum for $0258+35$. a) VLA at $8.4 \mathrm{GHz}$, b) VLA at $22.5 \mathrm{GHz}$, c) VLBA at $1.6 \mathrm{GHz}$, d) spectrum. Contours are traced at $(1,2,4, \ldots)$ times the $3 \sigma$ noise level; observational parameters (beam size, noise level, and peak brightness) are given in Table 3 . The cross in the VLA images indicates the position of the VLBA core. The integrated spectrum is derived from data in the present work and from the literature (see Sect. 3).

the presence of a surrounding low brightness extended structure in the VLA images. We note that in this source the inner jet direction appears to be constant, moreover no amount of bending is visible in the NW lobe, therefore the source structure on the large scale should be related not to the inner BH motion but to interaction with the ISM. We note that the estimated radiative age should imply a larger source size even allowing for a low lobe advance velocity.

\section{3. $0648+27$}

This object $\left(z=0.0414\right.$, corresponding to $0.82 \mathrm{kpc} /{ }^{\prime \prime}$, $\left.P_{408 \mathrm{MHz}}=10^{24.02} \mathrm{~W} \mathrm{~Hz}^{-1}\right)$ is only slightly extended at the lowest frequencies (Parma et al. 1986). It was resolved into a double source extended about $1^{\prime \prime}$ with VLA observations at $8.4 \mathrm{GHz}$ by Morganti et al. (2003); they also detect a large amount of HI using the Westerbork Synthesis Radio Telescope (WSRT, $M_{\mathrm{HI}}=1.1 \times 10^{10} M_{\odot}$ ). However, Morganti et al. (2003) are not able to identify a core and consequently interpret the radio structure in terms of a pair of symmetric lobes.

Our 8.4 GHz VLA data (Fig. 5, top left panel) confirm the structure as a double, with flux densities of 19 and $14 \mathrm{mJy}$ in the northern and southern components, respectively, in agreement with the data from Morganti et al. (2003). However, both components are resolved at $22 \mathrm{GHz}$ (see top right panel in Fig. 5), and a compact feature emerges in the north with a flux density of $2.5 \mathrm{mJy}$. Its small size $\left(<0.07^{\prime \prime}\right)$ and flatter spectral index suggest that this component is actually the core, with emission on either side. See Table 5 for a list of our data and spectral index measurements for the two lobes.

Our phase referenced VLBI data lend strong support to this scenario. In fact, the emission in the VLBA image is located in the vicinity of the VLA peak in the northern lobe. The total flux density is only $12.8 \mathrm{mJy}$, with a peak of $4.4 \mathrm{mJy} / \mathrm{beam}$. A faint jet-like structure is visible to the south-east, although the signal-to-noise is very poor and it could be spurious. In any case the difference between the total correlated flux and the peak flux density in the VLA image suggests the presence of some extended emission on intermediate scales. We note that the spectral index between the $22 \mathrm{GHz}$ VLA unresolved component and the total VLBA correlated flux is $\alpha_{1.6}^{22}=0.47 \pm 0.03$ (see Table 6). It is possible that this component is a jet knot and the core is at the extreme northern end. The core might be free-free or self-absorbed at $1.6 \mathrm{GHz}$, so that the VLBA does not pick out the center of activity. 

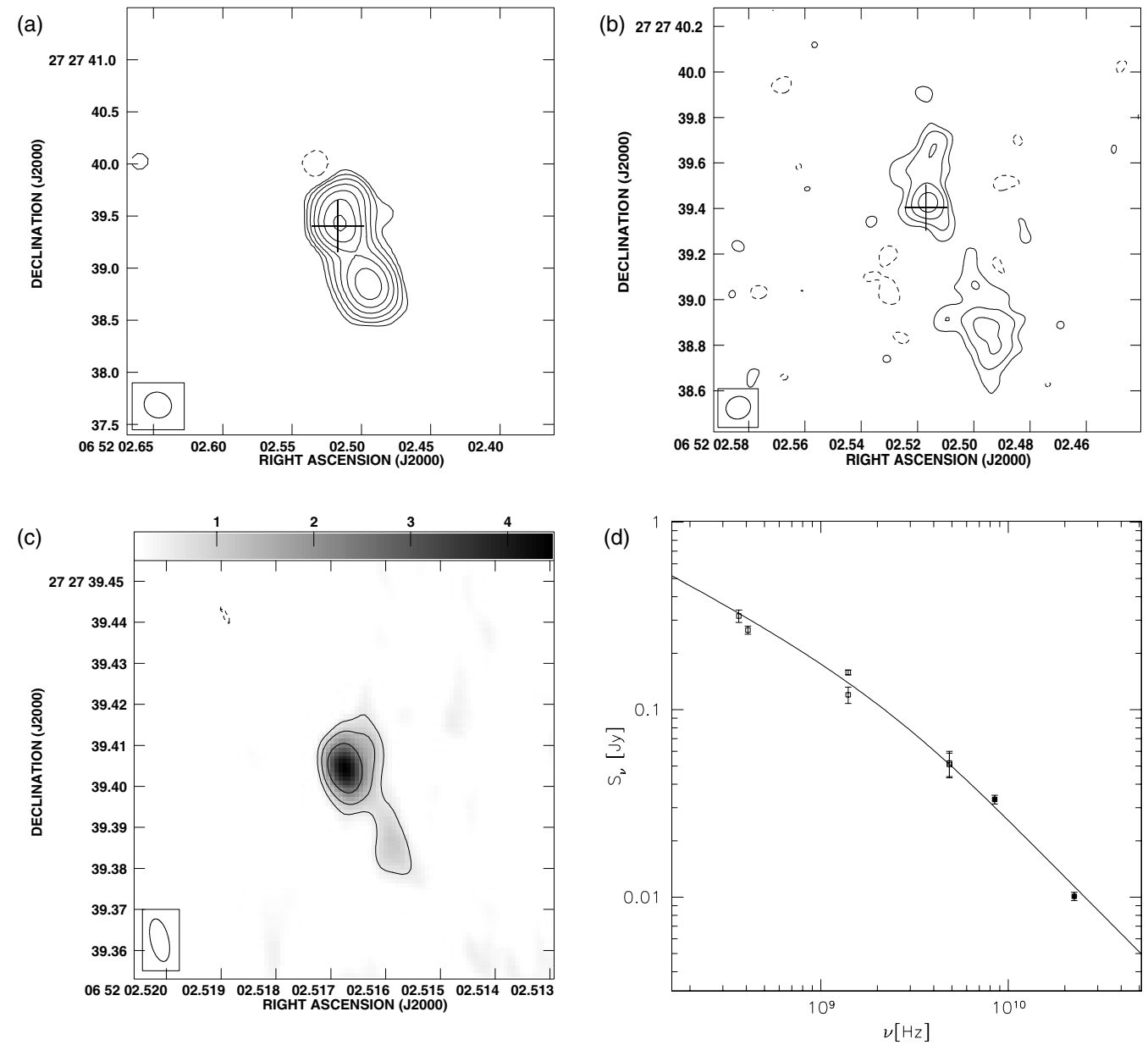

Fig. 5. Images and spectrum for $0648+27$. a) VLA at $8.4 \mathrm{GHz}$, b) VLA at $22.5 \mathrm{GHz}$, c) VLBA at $1.6 \mathrm{GHz}$, d) spectrum. Contours are traced at $(1,2,4, \ldots)$ times the $3 \sigma$ noise level; observational parameters (beam size, noise level, and peak brightness) are given in Table 3 . The cross in the VLA images indicates the position of the VLBA core. The integrated spectrum is derived from data in the present work and from the literature (see Sect. 3).

The source flux density is dominated by the extended emission. The total spectrum of the source between $325 \mathrm{MHz}$ and $22 \mathrm{GHz}$ has an index $\alpha \sim 0.8$, with a hint of steepening at high frequency $\left(\alpha_{8.4}^{22}=1.22 \pm 0.02\right)$. A measurement at $5 \mathrm{GHz}$ $\left(S_{5 \mathrm{GHz}}=213 \mathrm{mJy}\right.$, Antonucci 1985$)$ deviates significantly from the other measurements and was not included in the spectrum. If it is not due to an error, then it is difficult to understand this value.

The two lobes do not show any evidence for jet-like structure or the presence of hot spots. The lobe structure is relaxed with a relatively steep spectrum. The South lobe is at a greater distance with respect to the core, but we ascribe this asymmetry to a difference in the ISM and not to a relativistic effect. We note also that the lack of prominent jet structures in the parsec scale image suggests that no relativistic jet structure is present or that the source is on the plane of the sky. The faintness of the nuclear emission also argues against a Doppler boosted jet.

We have estimated the average equipartition magnetic field in this source to be $B_{\mathrm{eq}} \sim 95 \times 10^{-6}$ Gauss. Using the break frequency $(2.9 \mathrm{GHz})$ estimated from total flux density measures we can estimate a minimum age for this source of about $1 \mathrm{Myr}$ $\left(9.9 \times 10^{5}\right.$ years $)$. We expect that the external lobe regions are much older, confirming that this source is confined, and is expected to remain compact similar to NGC 4278 (Giroletti et al. 2005) despite its relatively higher total radio power and larger size. The connection between the small size of the radio emission and the presence of a major merger in this galaxy about $10^{9}$ years ago, with the presence of a large amount of $\mathrm{HI}$ in this galaxy (Morganti et al. 2003), is remarkable.

\section{4. $1037+30$}

Located at $z=0.0911\left(1^{\prime \prime}=1.70 \mathrm{kpc}\right), 1037+30$ has $P_{408 \mathrm{MHz}}=10^{25.37} \mathrm{~W} \mathrm{~Hz}^{-1}$. It is only slightly resolved at $1.4 \mathrm{GHz}$ (Fanti et al. 1986) and not detected in any VLBI observations to date (Giovannini et al. 2005). In the optical, 1037+30 is identified with the brightest galaxy in the cluster Abell 923.

Our VLA images are shown in the top panels of Fig. 6. The $8.4 \mathrm{GHz}$ image reveals an edge-brightened structure, with complex sub-structures: jets, and lobes with hot spots. In the $22 \mathrm{GHz}$ image only a point-like component, probably the core, and the resolved NW hot spot are evident. As in other objects, the accurate phase-referenced VLBI image provides 

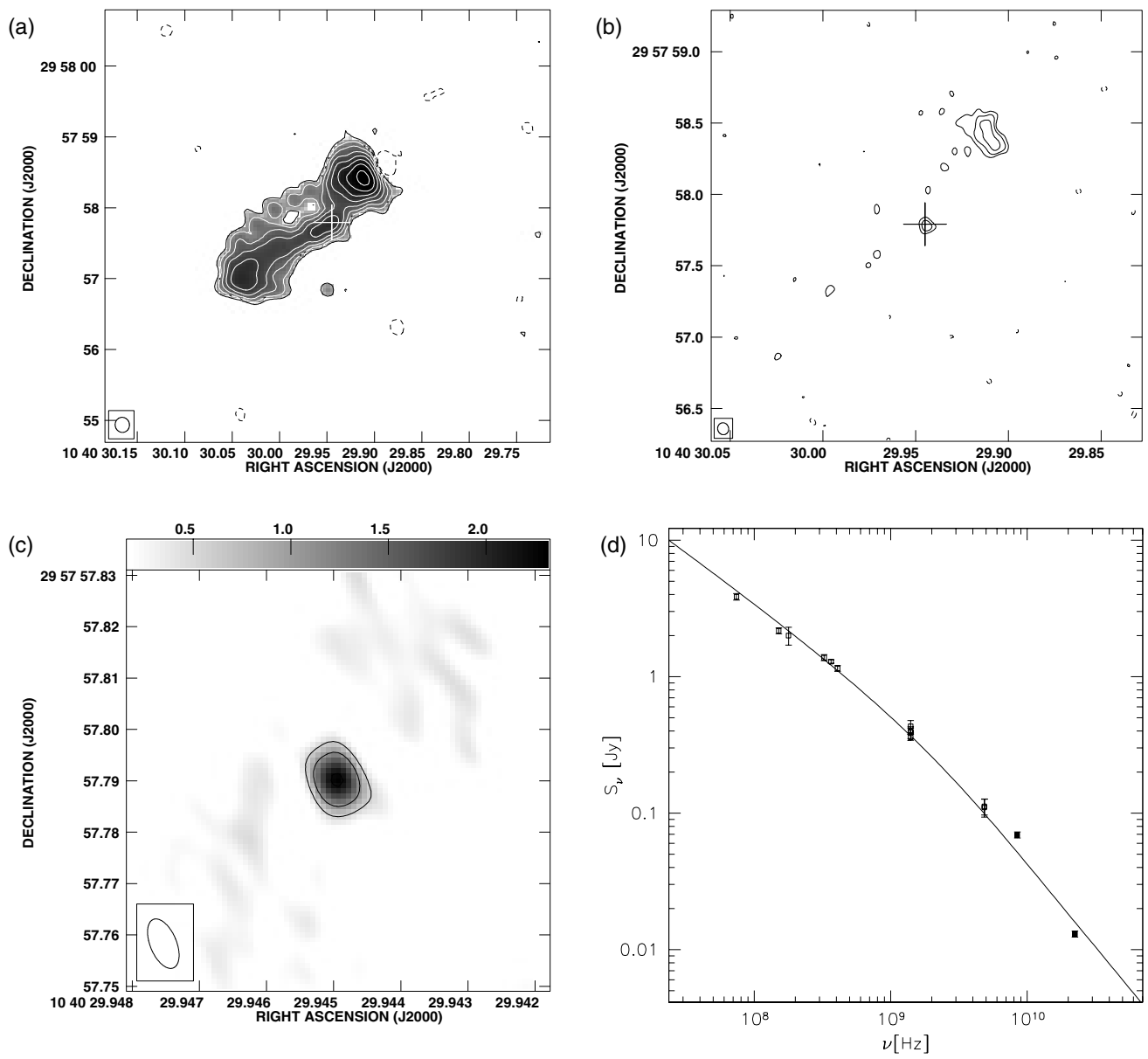

Fig. 6. Images and spectrum for $1037+30$. a) VLA at $8.4 \mathrm{GHz}$ (grey scale between 0.1 and $10 \mathrm{mJy} / \mathrm{beam}$ ), b) VLA at $22.5 \mathrm{GHz}$, c) VLBA at $1.6 \mathrm{GHz}, \mathbf{d})$ spectrum. Contours are traced at $(1,2,4, \ldots)$ times the $3 \sigma$ noise level; observational parameters (beam size, noise level, and peak brightness) are given in Table 3 . The cross in the VLA images indicates the position of the VLBA core. The integrated spectrum is derived from data in the present work and from the literature (see Sect. 3).

confirmation for the identification of the core which appears as a faint central component in the $8.4 \mathrm{GHz}$ image.

The south-eastern jet is clearly visible in the $8.4 \mathrm{GHz}$ VLA image, and is both longer and better defined than the jet in the opposite direction. While the jet brightness and length suggest that the SE is the approaching side, the NW hot spot is much brighter (with a peak of $13 \mathrm{vs} 2.3 \mathrm{mJy} /$ beam at $8.4 \mathrm{GHz}$ ). It is possible that the ISM density is irregular and that the brightness asymmetry is due to interactions with an inhomogeneous ISM.

The NW hot spot has a peculiar morphology, with a very sharp edge. We interpret this structure in terms of a back-flow due to a strong interaction of the NW jet with the ISM. This could also explain the compressed appearance of the NW hot spot, its higher brightness, and the "bridge" connecting the NW hot spot with the SE hot spot. Since the jet to counterjet brightness ratio in the $8.4 \mathrm{GHz}$ image is not high, we expect that the source is oriented at a large angle with respect to the line of sight. This orientation is in agreement with the non-detection of the jet at $22 \mathrm{GHz}$ and at $1.6 \mathrm{GHz}$ by the VLBA. This orientation is also consistent with the low core dominance, since relativistic jets at a large angles will have a Doppler factor $\ll 1$.

The VLBA data show a clear detection of a $4 \mathrm{mJy}$ core (see Table 6). Faint, diffuse emission is detected on the shortest baselines, although it is not possible to image it properly. At the very limit of the VLBA field-of-view, we detect radio emission from the north west hot spot region. Our image (Fig. 7) shows a resolved structure $\sim 60$ mas in size in agreement with the $22 \mathrm{GHz}$ image. The total flux density at $1.6 \mathrm{GHz}$ in this region is $\sim 40 \mathrm{mJy}$. Deeper images are necessary to see the connection to the jet.

The size and morphology of this source are in agreement with the definition of CSO sources. The jet interaction with the ISM is well defined. We estimated a dynamical age assuming a lobe expansion velocity of $0.2 \mathrm{c}$ and find an age of $4.5 \times 10^{4} \mathrm{yrs}$ assuming for the size the distance from the core of the SE hot spot. We do not estimate for this source a synchrotron age: the complex radio structure makes any estimate of an average $H_{\mathrm{eq}}$ unrealistic, and the contribution of different components to the total spectrum is difficult to weigh. The overall break frequency is however around $2.7 \mathrm{GHz}$.

We note that because of its total radio power we expect that $1037+30$ should evolve into an extended FR I radio galaxy. This source, like 0116+31 (4C 31.04, Giroletti et al. 2003), is also one of a number of low power radio sources with a clear CSO morphology. 


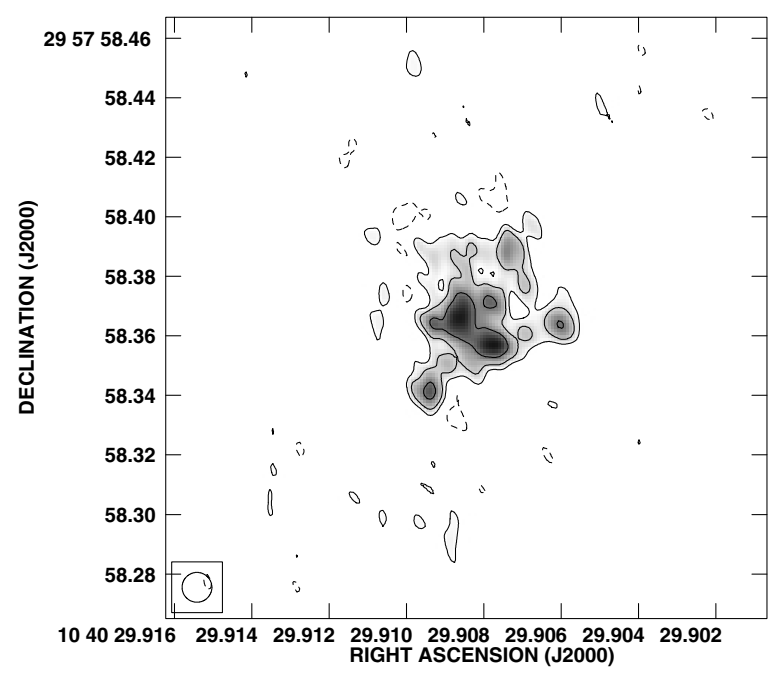

Fig. 7. Phase referenced VLBA image of the hot spot region of $1037+30$. Contours are traced at $(1,2,4,8, \ldots)$ times the $3 \sigma$ noise level. The lowest contour is $0.9 \mathrm{mJy} /$ beam and the peak is $5.6 \mathrm{mJy} / \mathrm{beam}$; the gray scale range is between 0.9 and $6 \mathrm{mJy} / \mathrm{beam}$. The beam is circular with a HPBW of 10 mas.

\section{5. $1855+37$}

Located at a redshift $z=0.0552\left(1^{\prime \prime}=1.07 \mathrm{kpc}\right), 1855+37$ has a total power $P_{408 \mathrm{MHz}}=10^{24.65} \mathrm{~W} \mathrm{~Hz}^{-1}$. Compact in the NVSS, this object is resolved into a triple source with higher resolution VLA observations at $1.4 \mathrm{GHz}$ (Fanti et al. 1986) and $5 \mathrm{GHz}$ (Morganti et al. 1997), where it also shows polarization. We identify $1855+37$ with the brightest member of the galaxy cluster CIZA J1857.6+3800, discovered on the basis of X-ray data by Ebeling et al. (2002) in the zone of avoidance. A discussion of the X-ray properties of $1855+37$ on the basis of ROSAT PSPC data is presented in Canosa et al. (1999) and Worrall \& Birkinshaw (2000).

Our observations at $8.4 \mathrm{GHz}$ (Fig. 8) resolve the flux density of $\sim 40 \mathrm{mJy}$ into a weak, diffuse emission extended over $\sim 7 "$. The structure is two sided, with a weak central component $\left(S_{\text {core }}=0.57 \mathrm{mJy}\right)$ and large, faint lobes. The source is not detected at $22 \mathrm{GHz}$; no significant peak is found in this region in the VLBA image.

Given the low power of the core, the lack of visible jets, and the rather steep spectral index, it is possible that the activity in this source is fading away. Note that in all previous observations, typically at lower frequency (Fanti et al. 1986; Morganti et al. 1997), the source was largely dominated by the extended lobes. For this reason, it was not possible to pinpoint the location of the nuclear activity, whose extreme weakness has therefore gone unnoticed. Our $8.4 \mathrm{GHz}$ observations reveal for the first time the exact position and flux density of the core, which is remarkably weak $\left(S_{\text {core }} / S_{\text {tot }}=0.017\right)$. This also explains the lack of detection of nuclear activity in $22 \mathrm{GHz}$ VLA data and in our VLBI observations. We note also that according to the correlation between the core and total radio power (see e.g. Giovannini et al. 2001) the nuclear power in this source is too low even assuming a large jet velocity and a huge Doppler de-boosting. We therefore propose this galaxy hosts a dying radio source. If the nucleus of this galaxy is ceasing its activity we would not expect that this source would significantly grow any larger than it is now $(\sim 7 \mathrm{kpc})$.

We note that the present radio structure is very similar to that found in NGC 4874 (the brightest galaxy in the Coma cluster) by Feretti \& Giovannini (1985). However, in NGC 4874 the presence of a core and of bright regions in the two lobes suggests ongoing nuclear activity.

The few data points available in the literature, combined with our new measurements at $8.4 \mathrm{GHz}$ and limit at $22.5 \mathrm{GHz}$, yield a break frequency $v_{\mathrm{br}} \sim 2.5 \mathrm{GHz}$. The equipartition magnetic field is $\sim 2.1 \times 10^{-5}$ Gauss and the corresponding radiative source age is $9.7 \mathrm{Myr}$. Since this source is inside an X-ray cluster we expect that its small size with respect to the radiative age can also be due to confinement from the IGM gas (see also Worrall \& Birkinshaw 2000). Also the source shape, similar to wide angle tail radio sources, could be due to relative motions between thermal gas and the source.

\section{Discussion}

We present new VLA and VLBA results for 5 Low Power Compact Radio Sources. Our high resolution images resolve all sources. The combined information of flat/inverted spectral indices $\left(-0.2 \lessgtr \alpha_{1.6}^{22} \lessgtr 0.5\right)$ and compactness unambiguously identify radio cores in four of them. In one case $(1855+37)$, we have a compact component at $8.4 \mathrm{GHz}$, which is a reasonable core candidate; however, a detection at another frequency would be desirable to confirm the classification. Most sources are symmetric, at least on arcsecond scales; $0222+36$ is also clearly symmetric on milliarcsecond scales. In general, the objects do not show any evidence for strong beaming effects (e.g. strong, one-sided jets, dominant cores). Thus, these sources are most likely close to the plane of the sky. We determine accurate values for their (projected) linear size, which must be, therefore, within a factor of a few from their intrinsic deprojected dimensions.

We show in Fig. 9 a radio power vs linear size diagram for the 95 sources in the Bologna Complete Sample (BCS). Radio power data are taken from the $\mathrm{B} 2$ survey at $408 \mathrm{MHz}$ via the online VizieR service ${ }^{1}$ (Ochsenbein et al. 2000) and linear sizes from several works in the literature ${ }^{2}$. The solid points denote the five LPC sources in the subsample considered in the present work with accurate linear size measurements. We confirm that this class of radio sources is characterized by a linear size < $10 \mathrm{kpc}$. The starred points indicate the two smallest sources in the BCS $(L S<0.1 \mathrm{kpc})$, one of which is a genuinely young

\footnotetext{
${ }^{1}$ http://vizier.u-strasbg.fr/viz-bin/VizieR

2 The largest part of the data is drawn from Fanti et al. (1987) and from "An Atlas of DRAGNs" for sources that are part of the 3CRR sample (see http://www.jb.man.ac.uk/atlas/); a few individual sources are better studied in other references: 0116+31 (4C 31.04, Giroletti et al. 2003), 0836+29B (Fanti et al. 1986), 1144+35 (Giovannini et al. 1999), 1217+29 (NGC 4278, Giroletti et al. 2005), $1257+28$ (NGC 4874, Feretti \& Giovannini 1985), 1557+26 (from the FIRST survey, Becker et al. 1995), 1652+39A (Mkn 501, Cassaro et al. 1999)
} 

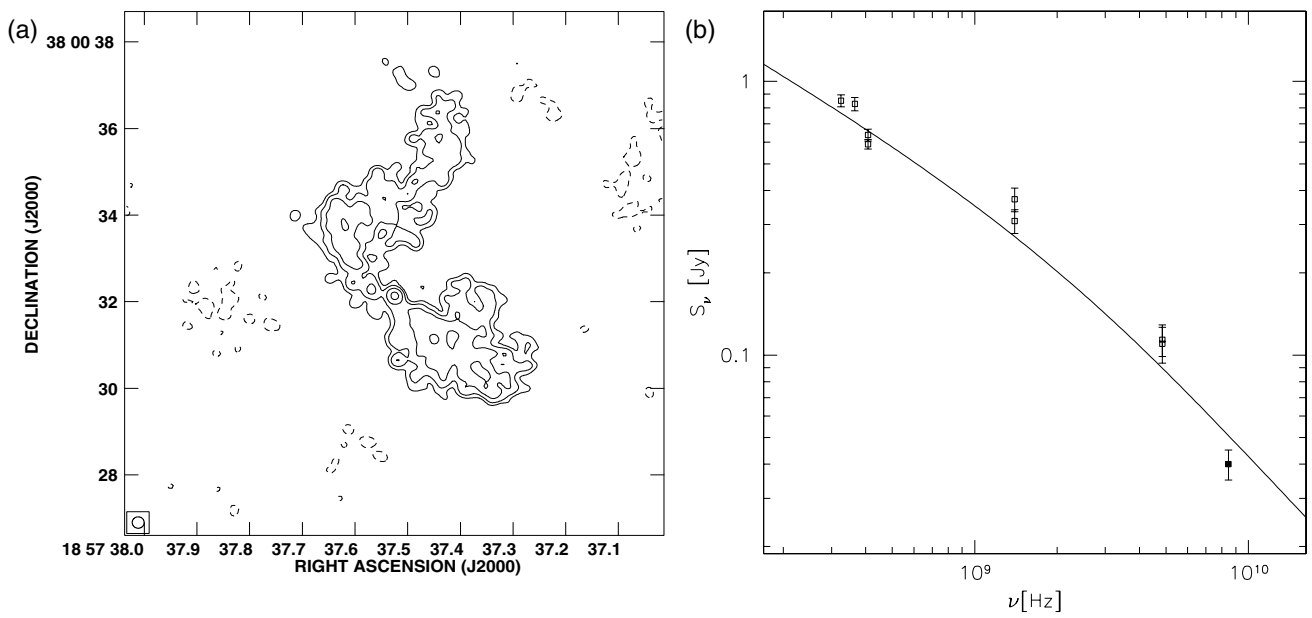

Fig. 8. The VLA image and spectrum for $1855+37$. a) VLA at $8.4 \mathrm{GHz}$, b) spectrum. Contours are traced at $(1,2,4, \ldots)$ times the $3 \sigma$ noise level; observational parameters (beam size, noise level, and peak brightness) are given in Table 3 . The source is not detected with the VLA at $22 \mathrm{GHz}$ nor at $1.6 \mathrm{GHz}$ with the VLBA. The integrated spectrum is derived from data in the present work and from the literature (see Sect. 3).

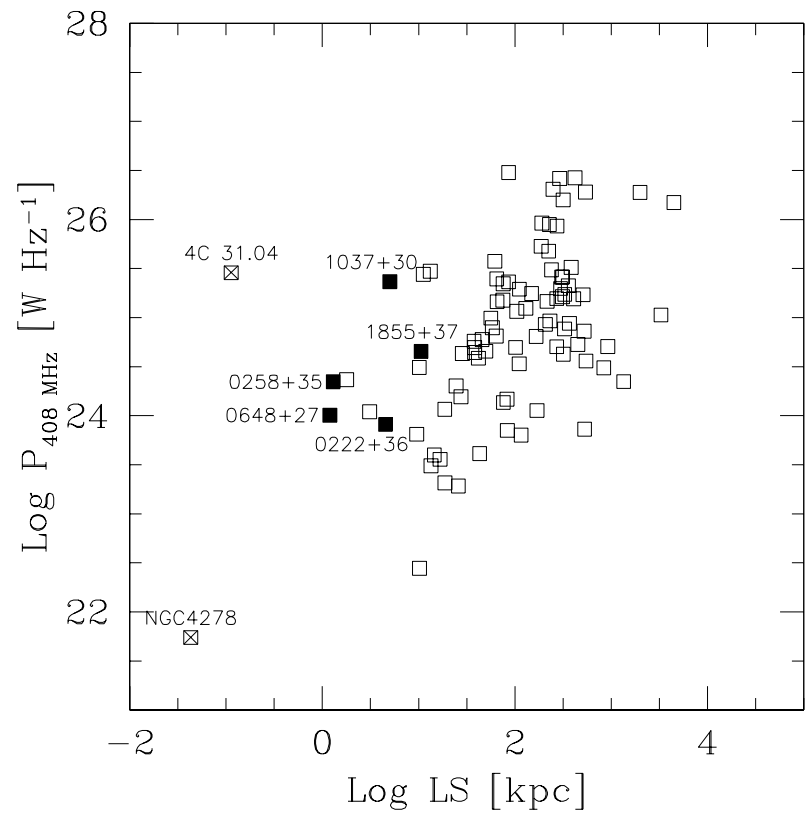

Fig. 9. Radio power vs. linear size diagram for sources in the Bologna Complete Sample (BCS, Giovannini et al. 2005). The filled squares indicate sources studied in the present work, while the starred symbols are for the two other compact sources in the sample: $0116+31$ (4C 31.04, Giroletti et al. 2003) and 1217+29 (NGC 4278, Giroletti et al. 2005)

CSO (4C 31.04, Giroletti et al. 2003), while the other is associated with a less active LINER galaxy (NGC 4278, Giroletti et al. 2005). From the distribution shown in the diagram of Fig. 9, we note that the small size of LPCs is not related to the low power of the radio source. In fact, there is also a large number of extended $\left(L S>10^{2} \mathrm{kpc}\right)$ radio galaxies with a similarly low radio power.

Our new LPC sources are more extended than classical young radio sources like the CSOs (Gugliucci et al. 2005), and could represent the intermediate age sources in between CSOs and classic kpc scale radio galaxies. The youth scenario has the problem of the lack of objects filling in the gap between sources with age $<10^{4} \mathrm{yrs}$ and $>10^{7} \mathrm{yrs}$; in particular, there seem to be too many bright young sources. However, this problem can be solved if the radio sources dim in luminosity as they grow up; for example, assuming equipartition conditions and expansion losses, Begelman (1996) reconciles the observational data with a model that predicts that the radio luminosity should decrease with size, roughly as $P_{\mathrm{R}} \propto L S^{-1 / 2}$. According to the evolution scenario young radio sources are then expected to be small and quite luminous.

In order to better understand radio source evolution and the radio power vs linear size diagram, we need to know the properties of compact radio sources. It is also important to point out that the constraints posed by the number counts per linear size range may be misleading. In fact, a number of explanations have been invoked, for example contamination from core-jet Doppler-boosted sources (Tinti et al. 2005) and a significant fraction of either frustrated or short lived sources (Readhead et al. 1994; Gugliucci et al. 2005).

To investigate these possibilities we are studying in detail compact (LS < $10 \mathrm{kpc}$ ) sources in our BCS, which are typically low power ones. If we consider the data presented here for 5 sources and include also the two other sources previously studied (4C 31.04, NGC 4278; Giroletti et al. 2003, 2005), we are presented with a variety of behaviors. As a matter of fact, the individual study of each source suggests that all cases are possible and we have evidence in a number of cases that sources are not able to grow because of an underpowered core or a jet instability, or because they are dying. On the other hand, we still have only a few cases in which there is clear evidence of a source which is growing, with significant interaction between the jet and the ISM. In particular, among sources studied here, we have:

- one source with evidence of a growing jet structure interacting with the ISM $(1037+30)$ with an estimated kinematic age of $5 \times 10^{4} \mathrm{yrs}$;

- a peculiar source in which an old structure and a younger one coexist $(0222+36$, in which the estimated radiative age 
of the halo is $\sim 10^{8} \mathrm{yrs}$, while the lobes are $10^{4}-10^{5} \mathrm{yrs}$ old). We interpret this structure as due to a jet instability which does not allow it to create large scale lobes. The old halo is permeated by relativistic electrons which slowly escape from the younger region (lobes) and diffuse into the surrounding volume;

- three sources that are small because they are short lived or frustrated. In $0258+35$, which has no hot spots and resembles a FR I radio galaxy, the VLA structure does not show any evidence of interaction between expanding jets and the ISM. The VLBA structure (a faint core and an isolated more powerful knot) suggests variable levels of activity. In $0648+27$ and $1855+37$ we find a low power nuclear region and little or weak extended emission, suggesting that they are possibly frustrated and/or dying. In the HI rich $0648+27$, we detected a nuclear source with the VLBA and the estimated radiative age is $\sim 10^{5} \mathrm{yrs}$, while in $1855+37$ the nuclear source is no longer active and the source radiative age is $10^{7} \mathrm{yrs}$. In general, the dynamic ages estimated from their large-scale structure and assuming typical lobe advance velocities are about one order of magnitude lower than the spectral estimates. Unless there is some misleading assumption (e.g., equipartition conditions do not apply), the advance velocities ( $\ll 0.1 c$ ) have to be significantly lower than those measured in CSOs.

\section{Conclusions}

Despite the poor statistics, our preliminary studies indicate that multiple causes can produce sources in the LPC class. In addition to flat or inverted spectrum sources dominated by projection effects as BL-Lacs, a small size can stem from: youth (4C 31.04 and 1037+30), instabilities in the jets (in space, as in $0222+36$, or time, as in $0258+35)$, frustration $(0648+27)$, a premature end of nuclear activity $(1855+37)$, or just a very low power core (NGC 4278). A more detailed discussion will appear in a future paper about the BCS. The study of the BCS, as a well defined complete sample, will allow us to make a statistical study to derive the source evolution. From the number of sources in different evolutionary stages it should be possible to estimate the duration and probability of different stages in radio source life.

Acknowledgements. We thank the referee Dr. C. Stanghellini for a prompt and useful report. The National Radio Astronomy Observatory is a facility of the National Science Foundation operated under cooperative agreement by Associated Universities, Inc. This research has made use of the NASA/IPAC Extragalactic Database (NED) which is operated by the Jet Propulsion Laboratory, Caltech, under contract with NASA and of NASA's Astrophysics Data System (ADS) Bibliographic Services. This material is based upon work supported by the Italian Ministry for University and Research (MIUR) under grant COFIN 2003-02-7534.

\section{References}

Antonucci, R. R. J. 1985, ApJS, 59, 499

Becker, R. H., White, R. L., \& Helfand, D. 1995, ApJ, 450, 559

Begelman, M. C. 1996, Cygnus A - Study of a Radio Galaxy, 209
Blundell, K. M., Rawlings, S., \& Willott, C. J. 1999, AJ, 117, 677

Canosa, C. M., Worrall, D. M., Hardcastle, M. J., \& Birkinshaw, M. 1999, MNRAS, 310, 30

Capetti, A., Celotti, A., Chiaberge, M., et al. 2002, A\&A, 383, 104

Cassaro, P., Stanghellini, C., Bondi, M., et al. 1999, A\&AS, 139, 601

Dallacasa, D., Stanghellini, C., Centonza, M., \& Fanti, R. 2000, A\&A, 363, 887

Ebeling, H., Mullis, C. R., \& Tully, R. B. 2002, ApJ, 580, 774

Fanaroff, B. L., \& Riley, J. M. 1974, MNRAS, 167, 31P

Fanti, C., \& Fanti, R. 2003, ASP Conf. Ser., 300, 81

Fanti, C., Fanti, R., de Ruiter, H. R., \& Parma, P. 1986, A\&AS, 65, 145

Fanti, C., Fanti, R., de Ruiter, H. R., \& Parma, P. 1987, A\&AS, 69, 57

Fanti, R., Fanti, C., Schilizzi, R. T., et al. 1990, A\&A, 231, 333

Fanti, C., Fanti, R., Dallacasa, D., et al. 1995, A\&A, 302, 317

Feretti, L., \& Giovannini, G. 1985, A\&A, 147, L13

Ghisellini, G., \& Celotti, A. 2001, A\&A, 379, L1

Giovannini, G., Taylor, G. B., Arbizzani, E., et al. 1999, ApJ, 522, 101

Giovannini, G., Cotton, W. D., Feretti, L., Lara, L., \& Venturi, T. 2001, ApJ, 552, 508

Giovannini, G., Taylor, G. B., Feretti, L., et al. 2005, ApJ, 618, 635

Giroletti, M., Giovannini, G., Taylor, G. B., et al. 2003, A\&A, 399, 889

Giroletti, M., Giovannini, G., Feretti, L., et al. 2004, ApJ, 600, 127

Giroletti, M., Taylor, G. B., \& Giovannini, G. 2005, ApJ, 622, 178

Gugliucci, N. E., Taylor, G. B., Peck, A. B., \& Giroletti, M. 2005, ApJ, 622, 136

Ho, L. C., Filippenko, A. V., \& Sargent, W. L. W. 1997, ApJS, 112, 315

Hummel, E., Beck, R., \& Dettmar, R.-J. 1991, A\&AS, 87, 309

Kaiser, C. R., Dennett-Thorpe, J., \& Alexander, P. 1997, MNRAS, 292, 723

Kunert-Bajraszewska, M., Marecki, A., \& Spencer, R. E. 2004, European VLBI Network on New Developments in VLBI Science and Technology, 73

Lara, L., Giovannini, G., Cotton, W. D., et al. 2004, A\&A, 421, 899

Ledlow, M. J., \& Owen, F. N. 1996, AJ, 112, 9

Marecki, A., Spencer, R. E., \& Kunert, M. 2003, Publications of the Astronomical Society of Australia, 20, 46

Marscher, A. P. 1987, Superluminal Radio Sources, 280

Morganti, R., Parma, P., Capetti, A., et al. 1997, A\&AS, 126, 335

Morganti, R., Oosterloo, T. A., Capetti, A., et al. 2003, A\&A, 399, 511

Murgia, M., \& Fanti, R. 1996, Rapporto Interno IRA, 228/96

Murgia, M., Fanti, C., Fanti, R., et al. 1999, A\&A, 345, 769

Narayan, R., \& Yi, I. 1995, ApJ, 452, 710

Ochsenbein, F., Bauer, P., \& Marcout, J. 2000, A\&AS, 143, 23

O'Dea, C. P. 1998, PASP, 110, 493

O’Dea, C. P., \& Baum, S. A. 1997, AJ, 113, 148

Owsianik, I., \& Conway, J. E. 1998, A\&A, 337, 69

Parma, P., de Ruiter, H. R., Fanti, C., \& Fanti, R. 1986, A\&AS, 64, 135

Polatidis, A. G., Conway, J. E., \& Owsianik, I. 2002, Proc. of the 6th EVN Symp., 139

Readhead, A. C. S., Xu, W., Pearson, T. J., Wilkinson, P. N., \& Polatidis, A. G. 1994, Compact Extragalactic Radio Sources, 17

Salvati, M., Spada, M., \& Pacini, F. 1998, ApJ, 495, L19

Sanghera, H. S., Saikia, D. J., Luedke, E., et al. 1995, A\&A, 295, 629

Tinti, S., Dallacasa, D., de Zotti, G., Celotti, A., \& Stanghellini, C. 2005, A\&A, 432, 31

Urry, C. M., \& Padovani, P. 1995, PASP, 107, 803

Wilkinson, P. N., Polatidis, A. G., Readhead, A. C. S., Xu, W., \& Pearson, T. J. 1994, ApJ, 432, L87

Worrall, D. M., \& Birkinshaw, M. 2000, ApJ, 530, 719 